\title{
Entanglement and relative entropy of a chiral fermion on the torus
}

\author{
Pascal Fries $\circledast^{1, *}$ and Ignacio A. Reyes ${ }^{2, \dagger}$ \\ ${ }^{1}$ Fakultät für Physik und Astronomie, Julius-Maximilians Universität Würzburg, \\ Am Hubland, 97074 Würzburg, Germany \\ ${ }^{2}$ Max-Planck-Institut für Gravitationsphysik, Am Mühlenberg 1, 14476 Potsdam, Germany
}

(Received 12 July 2019; published 19 November 2019)

\begin{abstract}
We derive the entanglement entropy of chiral fermions on the circle at arbitrary temperature. The spinsector contribution depends only on the total length of the entangling region, regardless of the configuration of the intervals. Thus, three-partite information provides a global indicator for the spin boundary conditions. Together with the modular Hamiltonian, our results provide a systematic way of obtaining relative entropy on the torus.
\end{abstract}

DOI: $10.1103 /$ PhysRevD.100.105015

\section{INTRODUCTION}

Entanglement is perhaps the most characteristic feature of quantum theory. Although there exist many measures for it, the most important one is the entanglement entropy. Given a global state defined via a density matrix $\rho$ and a subsystem $A$ of it, the reduced density matrix on $A$ is defined as $\rho_{A}:=\operatorname{tr}_{B}(\rho)$, where $B$ is the complement of $A$. Then, the entanglement entropy between $A$ and $B$ is simply the von Neumann entropy of the subsystem,

$$
S(A)=-\operatorname{Tr}_{A}\left[\rho_{A} \log \rho_{A}\right] .
$$

In practice, however, computing the logarithm in (1) is generically very challenging. Therefore, it is customary in QFT to resort to the replica trick. This consists of first computing a closely related quantity-the Rényi entropy for integer index $n$ :

$$
S_{n}(A)=\frac{1}{1-n} \log \operatorname{Tr} \rho_{A}^{n} .
$$

This requires computing the partition function of the theory on the $n$-fold cover of the manifold of interest, where the $n$ copies are glued cyclically along the entangling region $A$. In order for this strategy to work, it is crucial that we are able to find the analytic continuation of the Rényi entropy (2) away from integer $n$ and take the limit $n \rightarrow 1$. To the best of our knowledge, such analytic continuation

\footnotetext{
pascal.fries@physik.uni-wuerzburg.de ignacio.reyes@aei.mpg.de
}

Published by the American Physical Society under the terms of the Creative Commons Attribution 4.0 International license. Further distribution of this work must maintain attribution to the author(s) and the published article's title, journal citation, and DOI. Funded by SCOAP ${ }^{3}$. for the entanglement entropy of free fermions on the torus (finite temperature on the circle) is not known for an arbitrary torus modulus.

In this paper, we derive the entanglement entropy of the chiral fermion on the torus via a different route-by computing the entropy directly from the resolvent. This avoids the need to find the analytic continuation of the Rényi entropies. For the free fermion on the circle at finite temperature, the Rényi entropies for integer $n$ were first computed in Ref. [1] for a single interval and in Ref. [2] for multiple intervals. The analytic continuation to the entanglement entropy, however, was obtained for the high and low temperature expansions only.

The resolvent for the chiral fermion on the torus was recently obtained in Ref. [3] and also in Ref. [4]. For direct applications of the resolvent and modular Hamiltonian, we study entanglement entropy, mutual information, threepartite information, and relative entropy. Interestingly, while three-partite information vanishes for fermions in the plane [5], it is generally nonzero on the torus. Instead, we find that it is a global indicator of the boundary conditions, in the sense that its value does not depend on the precise configuration of the intervals involved.

The paper is organized as follows. In Sec. II, we review the resolvent method, the main tool that we will apply to later computations. In Sec. III, we go straight to the calculation of the entanglement entropy and discuss the mutual information. In Sec. IV, we consider relative entropy and illustrate some properties taking a small interval expansion. We summarize and discuss our results in Sec. VI.

\section{REVIEW OF THE RESOLVENT}

Let us quickly summarize the definition of the resolvent and the main steps involved in its derivation for the 
fermions on the torus [3]. A global state $\rho$ for the system on a generic Riemann surface determines the equal-time correlation function $G(x, y):=\left\langle\psi(x) \psi^{\dagger}(y)\right\rangle$. The resolvent of the correlator is defined via

$$
R(\xi):=[G+\xi-1 / 2]^{-1},
$$

where we always leave the space-time dependence of $R$ implicit. In (3), the inverse must be understood in the sense of distributions, i.e., as integrated against regular test functions. Equation (3) thus involves solving an integral equation for $R$.

One of the central results of Ref. [3] is the resolvent of a single chiral fermion on the torus for an arbitrary number $p$ of disjoint intervals $A=\cup_{j=1}^{p}\left(a_{j}, b_{j}\right)$, with both $\nu=2$ (real periodic, complex antiperiodic) and $\nu=3$ (doubly antiperiodic) boundary conditions. We shall not focus on its derivation but rather quickly review its structure and explain its properties. Explicitly, it is given by

$$
R(\xi)=\frac{\delta(x-y)}{\xi-1 / 2}-\frac{F(x, y ; \xi)}{(\xi-1 / 2)^{2}},
$$

where $x, y \in A$ are any two points on the entangling region, and

$$
F(x, y ; \xi)=\frac{\xi-1 / 2}{\xi+1 / 2} G_{\nu}(x-y \mid \tau, L h)\left[\frac{\Omega(x \mid \tau)}{\Omega(y \mid \tau)}\right]^{\mathrm{i} h},
$$

where each term is defined as

$$
\begin{aligned}
h & =\frac{1}{2 \pi} \log \frac{\xi+1 / 2}{\xi-1 / 2} \\
G_{\nu}(z \mid \tau, \mu) & =\frac{\eta^{3}(\tau)}{\mathrm{i} \vartheta_{1}(z \mid \tau)} \frac{\vartheta_{\nu}(z-\mathrm{i} \mu \mid \tau)}{\vartheta_{\nu}(-\mathrm{i} \mu \mid \tau)}, \\
\Omega(x \mid \tau) & =-\prod_{j=1}^{p} \frac{\vartheta_{1}\left(x-a_{j} \mid \tau\right)}{\vartheta_{1}\left(x-b_{j} \mid \tau\right)} .
\end{aligned}
$$

Here, $L=\sum_{j}\left|b_{j}-a_{j}\right|$ is the total length of all intervals, and our conventions for the Dedekind eta and the Jacobi elliptic functions are described in the Appendix. Note the appearance of $G_{\nu}(z \mid \tau, L h)$, which is the propagator on the torus with a chemical potential $\mu=L h$ as we will show in Sec. V. As usual, we fix by convenience the periods of the torus to be $1, \tau$-physics on the torus depends only on $\tau$, and we can always recover the result for a different spatial circle by rescaling. We shall restrict to purely imaginary modulus $\tau=\mathrm{i} \beta$ where $\beta$ is the inverse temperature, keeping in mind that the general case can be recovered by analytic continuation.

The knowledge of the resolvent was further used to derive the modular Hamiltonian for an arbitrary number of intervals on the torus. The modular Hamiltonian $K$ of a density matrix is defined via

$$
\rho=\frac{\mathrm{e}^{-K}}{\operatorname{Tre}^{-K}}
$$

and has found numerous applications in many-body quantum systems [6-8], quantum information [9-11], quantum field theory [12-17], modular theory [18,19], and the AdS/CFT correspondence [20-25].

The modular Hamiltonian for the chiral fermion on the torus exhibits a surprisingly interesting structure. Even for a single interval, the modular flow couples any given point to an infinite but discrete set of other points. These accumulate near the boundaries of the interval, where their contribution becomes increasingly damped or "redshifted" as they approach the end points. In the limit of zero temperature, these points "condense" regularly in the interval, giving rise to continuous nonlocality [3].

\section{ENTANGLEMENT ENTROPY}

We now determine the entanglement entropy on the torus of an arbitrary set of disjoint intervals. As mentioned above, once the resolvent is obtained, the entanglement entropy follows by a trace formula [12]:

$$
S=-\operatorname{Tr} \int_{1 / 2}^{\infty} \mathrm{d} \xi\left[(\xi-1 / 2)[R(\xi)-R(-\xi)]-\frac{2 \xi}{\xi+1 / 2}\right] .
$$

Replacing the resolvent (4), one finds

$S=\int_{A} \mathrm{~d} x \lim _{y \rightarrow x} \int_{1 / 2}^{\infty} \mathrm{d} \xi(\xi-1 / 2)\left[\frac{F(\xi)}{(\xi-1 / 2)^{2}}-\frac{F(-\xi)}{(\xi+1 / 2)^{2}}\right]$.

Next, we substitute $F$ from (5), and the integrand becomes

$$
\frac{1}{\xi+1 / 2} \lim _{y \rightarrow x}\left[G_{\nu}(x-y \mid \tau, L h)\left[\frac{\Omega(x \mid \tau)}{\Omega(y \mid \tau)}\right]^{\mathrm{i} h}-(h \rightarrow-h)\right] .
$$

In the limit $y \rightarrow x, G(x-y \mid \tau, L h)$ diverges like the UV propagator $1 / 2 \pi \mathrm{i}(x-y)$, but this leading divergence cancels in the above difference, leaving a well-defined expression. To extract the finite contribution, we use the Laurent series (B1) and (B2) provided in the Appendix. Then, the integrand in (9) is

$$
\frac{1}{\xi+1 / 2}\left[\frac{h}{\pi} \partial_{x} \log \Omega(x \mid \tau)+2 \Sigma_{\nu}(\Lambda \mid \tau)\right] .
$$

Here, the first term is position dependent but identical for each spin sector, whereas the second term is spin dependent but spatially constant. It is given by a Laurent expansion 


$$
\begin{aligned}
& \Sigma_{3}(\Lambda \mid \tau)=\sum_{\substack{k \geq 1 \\
k \text { odd }}}\left[\frac{q^{k}}{\Lambda^{-1}+q^{k}}-\frac{q^{k}}{\Lambda+q^{k}}\right], \\
& \Sigma_{2}(\Lambda \mid \tau)=\frac{1}{2} \frac{\Lambda-1}{\Lambda+1}+\sum_{\substack{k \geq 2 \\
k \text { even }}}\left[\frac{q^{k}}{\Lambda^{-1}+q^{k}}-\frac{q^{k}}{\Lambda+q^{k}}\right],
\end{aligned}
$$

where we used the convenient variable

$$
\Lambda=\mathrm{e}^{2 \pi L h}=\left[\frac{\xi+1 / 2}{\xi-1 / 2}\right]^{L}
$$

and $q=\mathrm{e}^{\mathrm{i} \pi \tau}$.

Thus, we learn from (10) that the entanglement entropy decomposes into a spin-independent part $S^{(0)}$ and a spindependent one $S^{(\nu)}$,

$$
S=S^{(0)}+S^{(\nu)}
$$

In the next subsections, we consider them separately.

\section{A. Spin-independent entropy}

We start by rederiving the known results from Refs. [1,2]. Let us start by focusing on the first contribution in (10), which does not depend on the boundary conditions. Introducing it back into (9), the two integrals decouple as

$$
S^{(0)}=\frac{1}{2 \pi^{2}} \int_{1 / 2}^{\infty} \mathrm{d} \xi \frac{\log \frac{\xi+1 / 2}{\xi-1 / 2}}{\xi+1 / 2} \int_{A} \mathrm{~d} x \partial_{x} \log \Omega(x \mid \tau) .
$$

We see that only boundary terms from the spacial integral contribute, one per each end point of the intervals. This gives the final result for the spin-independent entropy

$$
\begin{aligned}
S^{(0)}= & \frac{1}{6}\left[\sum_{i, j} \log \left|\vartheta_{1}\left(b_{i}-a_{j} \mid \tau\right)\right|\right. \\
& -\sum_{i<j} \log \left|\vartheta_{1}\left(b_{i}-b_{j} \mid \tau\right) \vartheta_{1}\left(a_{i}-a_{j} \mid \tau\right)\right| \\
& \left.-p \log \left|\vartheta_{1}(\epsilon \mid \tau)\right|\right]
\end{aligned}
$$

which takes a form very reminiscent of the one described in Ref. [12], but with respect to the Jacobi $\vartheta_{1}$ function. Note that we introduced a UV regulator $\epsilon>0$, integrating only within $\left(a_{i}+\epsilon, b_{i}-\epsilon\right)$, to avoid the infinite pileup of entanglement at the end points of each interval.

\section{B. Spin-dependent entropy}

In this section, we derive our first new result: the contribution to entanglement entropy that does depend on the choice of boundary conditions. As mentioned above, we restrict to the sectors $\nu=2,3$. Let us start with $\nu=3$ or (doubly antiperiodic), which has no zero mode. Plugging the second term of (10) back into (9), we have

$$
S^{(3)}=2 L \int_{1 / 2}^{\infty} \frac{\mathrm{d} \xi}{\xi+1 / 2} \Sigma_{3}(\Lambda \mid \tau) .
$$

Notice that we performed the trivial integral over the entangling region right away to yield a global prefactor of $L$, the total length of the regions. The remaining integral is, however, much more challenging, and finding a closed form goes beyond the scope of this paper. In order to bring it into a more explicit form, we can change the variable of integration to $\Lambda$, so that

$$
S^{(3)}=2 \sum_{\substack{k \geq 1 \\ \text { odd }}} \int_{1}^{\infty} \frac{d \Lambda}{\Lambda\left(\Lambda^{1 / L}-1\right)}\left[\frac{q^{k}}{\Lambda^{-1}+q^{k}}-\frac{q^{k}}{\Lambda+q^{k}}\right] .
$$

Notice the integral is completely regular since $L<1$, as we fixed the length of the spatial circle to unity. We have found no analytic expression for (14), but it can easily be dealt with numerically.

The case $\nu=2$ or (real periodic, complex antiperiodic) is special, for it has a zero mode. This manifests in the presence of the additional term in (12) and gives an entropy of

$$
\begin{aligned}
S^{(2)}= & \int_{1}^{\infty} \frac{\mathrm{d} \Lambda}{\Lambda\left(\Lambda^{1 / L}-1\right)} \\
& \times\left[\frac{\Lambda-1}{\Lambda+1}+2 \sum_{\substack{k \geq 2 \\
\text { even }}}\left(\frac{q^{k}}{\Lambda^{-1}+q^{k}}-\frac{q^{k}}{\Lambda+q^{k}}\right)\right],
\end{aligned}
$$

which can again be computed numerically.

The main result of this section is that the spin-dependent contribution to the entropy depends only on the total length of the entangling region-a feature also present for the Rényi entropies [2].

\section{Mutual and three-partite information}

The mutual information between two disjoint intervals $A$ and $B$ is another important information theory quantity. It it a measure of the correlation between two distributions, here the two reduced density matrices $\rho_{A}$ and $\rho_{B}$, and is defined via

$$
I(A, B):=S(A)+S(B)-S(A B) .
$$

From (13), the spin-independent part of the mutual information between two nonintersecting intervals $A=$ $\left(a_{1}, b_{1}\right)$ and $B=\left(a_{2}, b_{2}\right)$ is explicitly given by

$$
I(A, B)=\frac{1}{6} \log \left|\frac{\vartheta_{1}\left(a_{2}-a_{1} \mid \tau\right) \vartheta_{1}\left(b_{2}-b_{1} \mid \tau\right)}{\vartheta_{1}\left(b_{2}-a_{1} \mid \tau\right) \vartheta_{1}\left(b_{1}-a_{2} \mid \tau\right)}\right| .
$$

In Fig. 1, we plot this expression for different temperatures, as we continuously vary the separation between the two intervals. 


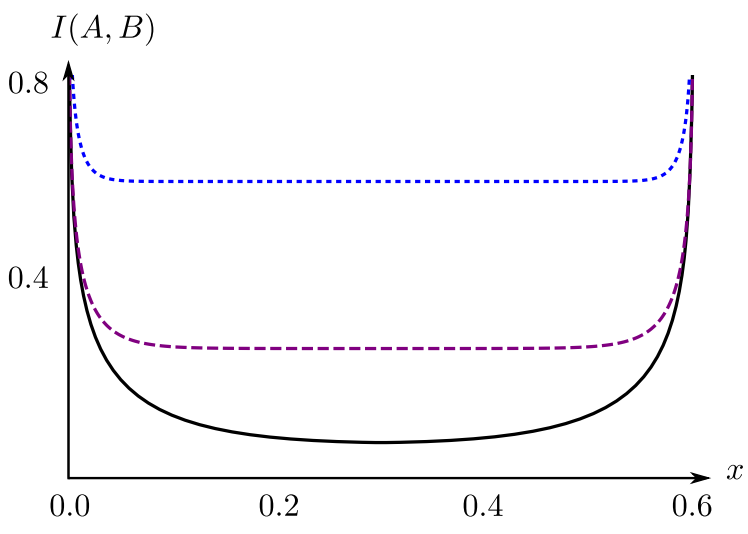

FIG. 1. Spin-independent part of the mutual information between the intervals $A=(0,0.2)$ and $B=(0.2+x, 0.4+x)$ as we vary their separation $x$, for $\beta=3$ (solid black), $\beta=0.16$ (dashed purple), and $\beta=0.07$ (dotted blue).

Remarkably, as pointed out in the previous section, the spin-dependent part to the entropy depends on the entangling region only via its total length $L$. Therefore, its contribution to the mutual information does not depend on the distance between the intervals but only on their size

$$
S^{(\nu)}(A)+S^{(\nu)}(B)-S^{(\nu)}(A B)=f\left(L_{A}, L_{B}\right) .
$$

This is one the main results of this paper; mutual information is insensible to the fermion boundary conditions up to a global term. This was also shown in Ref. [2] for the Rényi mutual information $I_{n}(A, B)$ for $n \geq 2 \in \mathbb{Z}$.

This property, together with the spin-independent entropy (13), implies that on the torus-in contrast to the plane [5] - the three-partite information [26]

$$
\begin{aligned}
I(A, B, C):= & S(A)+S(B)+S(C)-S(A B) \\
& -S(A C)-S(B C)+S(A B C)
\end{aligned}
$$

does not vanish but rather presents a global indicator for the boundary conditions, in the sense that it does not depend on the specific layout of the intervals.

\section{RELATIVE ENTROPY}

Another essential concept in information theory is relative entropy, also known as the Kullback-Leibler divergence. It is a measure of the distinguishability between two probability distributions $\rho$ and $\sigma$. Although it is not symmetric, it has a number of properties that make it fundamental due to its connection to many other information functions. It is defined by

$$
D(\rho \mid \sigma)=\operatorname{Tr}[\rho \log \rho]-\operatorname{Tr}[\rho \log \sigma] .
$$

Relative entropy is always positive and vanishes only if $\rho=\sigma$. As is well known, it can also be rewritten as

$$
D(\rho \mid \sigma)=\Delta\langle K\rangle-\Delta S
$$

in terms of the variations of the modular Hamiltonian $K_{\sigma}=$ $-\log \sigma-\log Z_{\sigma}$ and the entanglement entropy

$$
\begin{aligned}
\Delta\langle K\rangle & =\left\langle K_{\sigma}\right\rangle_{\rho}-\left\langle K_{\sigma}\right\rangle_{\sigma} \\
\Delta S & =S(\rho)-S(\sigma) .
\end{aligned}
$$

Here, $\sigma$ is the reference state since we used the modular Hamiltonian associated to $\sigma$ instead of $\rho$. The convenience of (18) is that presented in this form it closely resembles the first law of thermodynamics. Given that we have both the modular Hamiltonian from Ref. [3] and the entanglement entropy from Sec. III, we are in a position to compute the relative entropy directly using (18).

Consider the following class of density distributions. Given a global thermal state at some modulus $\tau$, the reduced density matrix on the subregion $A$ provides a reference state $\sigma=f(\tau)$. If we now start from a global state at some different modulus $\tau^{\prime}$ and reduce to the same region $A$, this will produce another state $\rho=f\left(\tau^{\prime}\right)$. We will consider the relative entropy between two such states.

We start by reviewing the structure of the modular Hamiltonian and then computing its expectation value. As referred to in the Introduction, an explicit expression of the modular Hamiltonian for chiral fermions on the torus was found in Ref. [3]. It contains a local and a bilocal term:

$$
K=K^{\mathrm{loc}}+K^{\mathrm{bi}-\mathrm{loc}} .
$$

As an operator, the local part is spin independent and takes the standard geometric form,

$$
K^{\mathrm{loc}}=\int_{A} \mathrm{~d} x \beta(x) T(x),
$$

where the stress tensor of the fermion is

$$
T(x)=\frac{\mathrm{i}}{2}\left[\psi^{\dagger} \partial_{x} \psi-\psi \partial \psi^{\dagger}\right](x)
$$

and $\beta(x)$ is known as the entanglement temperature, given by

$$
\beta(x)=\frac{2 \pi \beta}{2 \pi+\beta \partial_{x} \log \Omega(x \mid \tau)} .
$$

The entanglement temperature is the natural generalization of the more familiar Unruh temperature measured by an accelerated observer in the vacuum. Close to the end points of each interval, $\beta(x) \sim x$, and the modular Hamiltonian resembles that of Rindler space.

The bilocal term was the novel feature found for fermions on the torus. It couples a given point $x$ to an infinite but discrete set of other points $x_{k}(x)$ on the entangling region via 


$$
K_{ \pm}^{\mathrm{bi}-\mathrm{loc}}=\int_{A} \mathrm{~d} x \sum_{k \in \mathbb{Z}}( \pm 1)^{k} \tilde{\beta}\left(x, x_{k}(x)\right) \psi^{\dagger}(x) \psi\left(x_{k}(x)\right),
$$

where,+- correspond to the spin sectors $\nu=2,3$, respectively. Here, the points $x_{k}$ are solutions to the transcendental equation

$$
x-x_{k}+\beta g\left(x, x_{k}\right)-k=0
$$

with

$$
g(x, y)=\frac{1}{2 \pi L} \log \frac{\Omega(x \mid \tau)}{\Omega(y \mid \tau)}
$$

and $\Omega(z \mid \tau)$ as defined in (8). The bilocal entanglement temperature is

$$
\tilde{\beta}(x, y)=\frac{\mathrm{i} \pi}{L\left(1+\beta \partial_{x} g(x, y)\right) \sinh \pi g(x, y)} .
$$

Now, we consider the expectation value of each term in the modular Hamiltonian, which we will eventually replace back into the relative entropy (18). We start with the local term (19). Notice that the expectation value acts only on the operator $T$ in (20), while the entanglement temperature $\beta(x)$ is fixed by the reference state $\sigma$ (at modulus $\tau$ ) and remains the same for the perturbed state $\rho$. As expected, $\langle T\rangle$ is divergent due to operators evaluated at identical spacetime positions, but relative entropy is well defined since these UV divergences cancel in (18). Using the shorthand notation

$$
\Delta G_{\nu}(z)=G_{\nu}\left(z \mid \tau^{\prime}\right)-G_{\nu}(z \mid \tau),
$$

the variation of the local term reads

$$
\Delta\left\langle K^{\mathrm{loc}}\right\rangle=-\left.\mathrm{i} \partial_{z} \Delta G_{\nu}(z)\right|_{z=0} \int_{A} \mathrm{~d} x \beta(x) .
$$

Although we have not found a closed expression for the integral, it is easy to compute it numerically. Notice that, while $K^{\text {loc }}$ as an operator is independent of the spin sector, its expectation value is not. Indeed, the prefactor depends on the derivative of the propagator $G_{\nu}$. As we comment in the discussion, this implies that relative entropy is not spin independent.

On the other hand, the variation of the bilocal contribution is

$$
\begin{aligned}
\Delta\left\langle K^{\mathrm{bi}-\mathrm{loc}}\right\rangle= & \sum_{k \in \mathbb{Z}} \int_{A} \mathrm{~d} x( \pm 1)^{k} \tilde{\beta}\left(x, x_{k}(x)\right) \\
& \times \Delta G_{\nu}\left(x-x_{k}(x)\right) .
\end{aligned}
$$

This integral is technically difficult to deal with; one must first determine the solutions $x_{k}(x)$ to (21)-a transcendental equation involving elliptic functions-and then perform the integration.

Finally, the variation of the entanglement entropy also has two contributions,

$$
\Delta S=S\left(\tau^{\prime}\right)-S(\tau)
$$

Putting everything together, the relative entropy is given by

$$
D(\rho \mid \sigma)=\Delta\left\langle K^{\mathrm{loc}}\right\rangle+\Delta\left\langle K^{\mathrm{bi-loc}}\right\rangle-\Delta S^{(0)}-\Delta S^{(\nu)},
$$

where each term is given in (23), (24), (13), (14), and (15). As emphasized above, in practice, the evaluation of (25) is very challenging, and we leave it as future work.

\section{A. Example: The thermal cylinder}

In order to illustrate how to compute in principle this relative entropy, let us simplify things and perform the explicit calculation for a single interval of length $L$, in the limit when the size of the spatial circle is much larger than both the entangling region and the temporal cycle. We view this as an exercise combining known tools rather than a new result. Then, our expressions for the modular Hamiltonian and entanglement entropy on the torus reduce to the wellknown universal result on the thermal cylinder [14]

$$
\begin{aligned}
& K(\beta)=\beta \int_{0}^{L} \mathrm{~d} x \frac{\sinh \frac{\pi(L-x)}{\beta} \sinh \frac{\pi x}{\beta}}{\sinh \frac{\pi L}{\beta}} T(x), \\
& S(\beta)=\frac{1}{6} \log \left[\frac{\beta}{\pi \epsilon} \sinh \frac{\pi L}{\beta}\right],
\end{aligned}
$$

where $T(x)$ is again the fermionic stress tensor. Notice that in this case the modular flow is purely local. As described above, by varying the inverse temperature $\beta$ of the parent state, we get a one-parameter family of states defined on $A$. We can compute the relative entropies between such states.

For this simplified case, it is easy to obtain (23) from the fermionic propagator and find an explicit expression for the relative entropy:

$$
\begin{aligned}
D\left(\beta \mid \beta^{\prime}\right)= & \frac{\beta}{24}\left[\frac{1}{\beta^{\prime 2}}-\frac{1}{\beta^{2}}\right]\left[-\beta+\pi L \operatorname{coth} \frac{\pi L}{\beta}\right] \\
& +\frac{1}{6} \log \frac{\beta \sinh \frac{\pi L}{\beta}}{\beta^{\prime} \sinh \frac{\pi L}{\beta^{\prime}}} .
\end{aligned}
$$

Finally, it is illustrative to plot this function for a fixed reference $\beta=2$ and interval length $L=1$, while we vary $\beta^{\prime}$; see Fig. 2.

As can be seen from the figure, the distribution obtained from a parent state at low $\beta^{\prime}$ (high temperature) is very distinguishable from the finite temperature state. On the other hand, the relative entropy with respect to the vacuum $\beta^{\prime} \rightarrow \infty$ asymptotes to a constant. Relative entropy only 


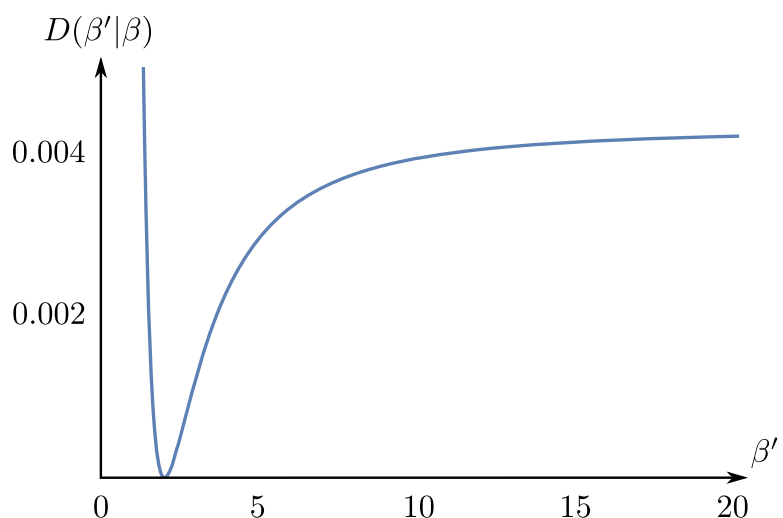

FIG. 2. Relative entropy between the states obtained by reducing a thermal state on the line at temperature $\beta^{\prime}$ and $\beta$, respectively, to a single interval of length $L=1$. The reference temperature associated to $\sigma$ is $\beta=2$.

vanishes at $\beta^{\prime}=\beta$, as it should, and the fact that the curve has vanishing slope at that point is the first law of entanglement. Again, we take this as a pedagogical first step in the explicit computation of relative entropy and leave for future work the more involved calculations on the torus.

\section{RESOLVENT WITH A CHEMICAL POTENTIAL}

In order to repeat the calculations for the two remaining cases $\nu=1,4$ (doubly periodic and real antiperiodic, complex periodic), let us derive the resolvent in these sectors. We will do this explicitly for $\nu=1$; the other case is entirely analogous. Consider first the Green's function

$$
G(z \mid \tau, \mu)=\sum_{k \in \mathbb{Z}} \frac{\mathrm{e}^{-2 \pi \mathrm{i} k z}}{1-\mathrm{e}^{2 \pi(\mathrm{i} k \tau-\mu)}},
$$

where we introduced a chemical potential $\mu$ to regularize the divergent term at $k=0$. The above series does not converge pointwise; however, it does converge in the sense of distributions: Starting from the geometric series

$$
\frac{1}{2 \mathrm{i}} \cot \pi z=\frac{1}{2} \frac{\mathrm{e}^{\mathrm{i} \pi z}+\mathrm{e}^{-\mathrm{i} \pi z}}{\mathrm{e}^{\mathrm{i} \pi z}-\mathrm{e}^{-\mathrm{i} \pi z}}=\frac{1}{2}+\sum_{k \geq 1} \mathrm{e}^{-2 \pi \mathrm{i} k z}
$$

for $\Im[z]<0$, we find

$$
\begin{aligned}
G(z \mid \tau, \mu)= & \frac{1}{2 \mathrm{i}} \cot \pi z-\frac{1}{2}+\frac{1}{1-\mathrm{e}^{-2 \pi \mu}} \\
& +\sum_{\substack{k \geq 2 \\
\text { even }}}\left[\frac{\mathrm{e}^{\mathrm{i} \pi k z}}{1-\mathrm{e}^{-2 \pi \mu} q^{-k}}+\frac{\mathrm{e}^{-\mathrm{i} \pi k z}}{1-\mathrm{e}^{-2 \pi \mu} q^{k}}-\mathrm{e}^{-\mathrm{i} \pi k z}\right],
\end{aligned}
$$

which can be rewritten as

$$
\begin{aligned}
G(z \mid \tau, \mu)= & \frac{1}{2 \mathrm{i}} \cot \pi z+\frac{1}{2} \frac{1+\mathrm{e}^{-2 \pi \mu}}{1-\mathrm{e}^{-2 \pi \mu}} \\
& -\sum_{\substack{k \geq 2 \\
\text { even }}}\left[\frac{\mathrm{e}^{\mathrm{i} \pi k z} q^{k}}{\mathrm{e}^{-2 \pi \mu}-q^{k}}-\frac{\mathrm{e}^{-\mathrm{i} \pi k z} q^{k}}{\mathrm{e}^{2 \pi \mu}-q^{k}}\right] .
\end{aligned}
$$

The right-hand side is now absolutely convergent on the strip $-\Im[\tau]<\Im[z]<\Im[\tau]$ and yields

$$
G_{\nu}(z \mid \tau, \mu)=\frac{\eta^{3}(\tau)}{\mathrm{i} \vartheta_{1}(z \mid \tau)} \frac{\vartheta_{\nu}(z-\mathrm{i} \mu \mid \tau)}{\vartheta_{\nu}(-\mathrm{i} \mu \mid \tau)}
$$

for general spin structure $\nu=1,2,3,4$.

As can be readily seen from the quasiperiodicities of the theta functions, Eq. (27) satisfies the generalized KuboMartin-Schwinger boundary condition

$$
G(z+\tau \mid \tau, \mu)= \pm \mathrm{e}^{-2 \pi \mu} G(z \mid \tau, \mu) .
$$

We can thus use the methodology of Ref. [3] to derive the resolvent as (4) with

$F(x, y ; \xi)=\frac{\xi-1 / 2}{\xi+1 / 2}\left[\frac{\Omega(x)}{\Omega(y)}\right]^{\mathrm{i} h} G(x-y \mid \tau, \mu+L h)$.

Note that, unlike the Green's function (27), the resolvent is well defined in the limit $\mu \rightarrow 0$.

Having found the resolvent, we can now in principle repeat our calculations for the cases $\nu=1$, 4. However, there is one caveat: because of the Bose statistics in (26), $G$ has an unbounded spectrum, and thus we have to find new valid formulas for the modular Hamiltonian, entanglement, and relative entropy in terms of the resolvent. While this is easy for the modular Hamiltonian, we have yet to find good expressions for the entropies.

\section{CONCLUSIONS}

In this short paper, we have described some applications of the modular Hamiltonian found in Ref. [3] for the chiral fermion on the circle at finite temperature. First, we found an exact expression for the entanglement entropy for multiple intervals, valid for any torus modulus $\tau$, i.e., generic spatial and thermal circles. This is a generalization of the results in Refs. [1,2], where the authors provided the entanglement entropy in the high and low temperature expansions. Whereas the spin-independent contribution to the entanglement entropy was known from these works, the novel result of this paper was to derive the analytic continuation of the spin-dependent term for an arbitrary torus.

Remarkably, this piece depends on the entangling intervals only via the total length of the region $L=\sum_{j}\left|b_{j}-a_{j}\right|$, and not the details of its configuration. This implies that the three-partite information of three 
intervals is a, generally nonvanishing, global indicator of the spin structure.

The third result of this paper is an explicit formula for the relative entropy $D(\rho \mid \sigma)$ of the free fermion on the torus. As the reference state, we chose the reduced density matrix $\sigma$ on multiple intervals coming from a global state on the torus of modulus $\tau=i \beta$. The target state is obtained in the same way, but starting from a different temperature $\beta^{\prime}$. This provides a one-parameter family of states for computing relative entropy. Although in practice the integrals involved in the computation are very challenging, mainly due to the nonlocal terms involved, the final expression (25) is explicit and can be investigated numerically.

Moreover, we also observed that, while both the modular Hamiltonian as an operator and the entanglement entropy separate into a universal and spin-dependent parts, this does not hold for the relative entropy. This is simply because the computation of relative entropy involves expectation values, which yields Green's functions in the modular Hamiltonians. Since relative entropy is a measure of the distinguishability of two distributions, this implies that fermions with some boundary conditions are more distinguishable than others.

Finally, we showed that the propagator of the fermion on the torus with a chemical potential allows us to compute the resolvent for the spin sectors $\nu=1,4$ which were not considered in Ref. [3]. Moreover, the propagatorlike term that appears in the resolvent corresponds precisely to the propagator with a chemical potential. This is due to the identical boundary conditions imposed in both problems.

\section{ACKNOWLEDGMENTS}

P.F. is financially supported by the DFG Project No. DFG HI 744/9-1. The work of I. R. is funded by the Gravity, Quantum Fields and Information group at Max-Planck-Institut für Gravitationsphysik (AlbertEinstein-Institut), which is generously supported by the Alexander von Humboldt Foundation and the Federal Ministry for Education and Research through the Sofja Kovalevskaja Award. I. R. also acknowledges the hospitality of Perimeter Institute, where part of this work was done.

\section{APPENDIX A: CONVENTIONS}

Our definition for the Dedekind eta function is

$$
\eta(\tau):=e^{\pi i \tau / 12} \prod_{k \geq 1}\left(1-q^{2 k}\right)
$$

in terms of

$$
q:=\mathrm{e}^{\mathrm{i} \pi \tau} .
$$

Furthermore, we define the Jacobi theta function by

$$
\vartheta_{3}(z \mid \tau):=\sum_{k \in \mathbb{Z}} q^{n^{2}} e^{2 \pi \mathrm{i} z}
$$

while the auxiliary thetas are given by

$$
\begin{aligned}
& \vartheta_{4}(z \mid \tau)=\vartheta_{3}(z+1 / 2 \mid \tau), \\
& \vartheta_{2}(z \mid \tau)=q^{1 / 4} \mathrm{e}^{\mathrm{i} \pi z} \vartheta_{3}(z+\tau / 2 \mid \tau), \\
& \vartheta_{1}(z \mid \tau)=-\mathrm{i} q^{1 / 4} \mathrm{e}^{\mathrm{i} \pi z} \vartheta_{4}(z+1 / 2+\tau / 2 \mid \tau) .
\end{aligned}
$$

\section{APPENDIX B: LAURENT SERIES OF THE PROPAGATOR}

The propagators appearing in (7) are given by the following Laurent series,

$$
\begin{aligned}
G_{3}(z \mid \tau, L h)= & \frac{1}{2 \mathrm{i} \sin \pi z} \\
& +\sum_{\substack{k \geq 1 \\
\text { odd }}}\left[\frac{\mathrm{e}^{\mathrm{i} \pi k} q^{k}}{\Lambda^{-1}+q^{k}}-\frac{\mathrm{e}^{-\mathrm{i} \pi k} q^{k}}{\Lambda+q^{k}}\right] \\
G_{2}(z \mid \tau, L h)= & \frac{1}{2 \mathrm{i}} \cot \pi z+\frac{1}{2} \frac{1}{\Lambda+1} \\
& +\sum_{\substack{k \geq 2 \\
\text { even }}}\left[\frac{\mathrm{e}^{\mathrm{i} \pi k} q^{k}}{\Lambda^{-1}+q^{k}}-\frac{\mathrm{e}^{-\mathrm{i} \pi k} q^{k}}{\Lambda+q^{k}}\right],
\end{aligned}
$$

where again $\Lambda=e^{2 \pi L h}$.
[1] T. Azeyanagi, T. Nishioka, and T. Takayanagi, Near extremal black hole entropy as entanglement entropy via AdS(2)/CFT(1), Phys. Rev. D 77, 064005 (2008).

[2] C. P. Herzog and T. Nishioka, Entanglement entropy of a massive Fermion on a torus, J. High Energy Phys. 03 (2013) 077.

[3] P. Fries and I. A. Reyes, companion Letter, Entanglement Spectrum of Chiral Fermions on the Torus, Phys. Rev. Lett. 123, 211603 (2019).
[4] D. Blanco and G. Prez-Nadal, Modular Hamiltonian of a chiral fermion on the torus, Phys. Rev. D 100, 025003 (2019).

[5] H. Casini and M. Huerta, Remarks on the entanglement entropy for disconnected regions, J. High Energy Phys. 03 (2009) 048.

[6] A. Chandran, M. Hermanns, N. Regnault, and B. A. Bernevig, Bulk-edge correspondence in entanglement spectra, Phys. Rev. B 84, 205136 (2011). 
[7] L. Vanderstraeten, M. Mariën, J. Haegeman, N. Schuch, J. Vidal, and F. Verstraete, Bridging Perturbative Expansions with Tensor Networks, Phys. Rev. Lett. 119, 070401 (2017).

[8] W. Zhu, Z. Huang, and Y.-c. He, Reconstructing entanglement Hamiltonian via entanglement eigenstates, Phys. Rev. B 99, 235109 (2019).

[9] H. Li and F. D. M. Haldane, Entanglement Spectrum as a Generalization of Entanglement Entropy: Identification of Topological Order in Non-Abelian Fractional Quantum Hall Effect States, Phys. Rev. Lett. 101, 010504 (2008).

[10] J. I. Cirac, D. Poilblanc, N. Schuch, and F. Verstraete, Entanglement spectrum and boundary theories with projected entangled-pair states, Phys. Rev. B 83, 245134 (2011).

[11] M. Dalmonte, B. Vermersch, and P. Zoller, Quantum simulation and spectroscopy of entanglement Hamiltonians, Nat. Phys. 14, 827 (2018).

[12] H. Casini and M. Huerta, Reduced density matrix and internal dynamics for multicomponent regions, Classical Quantum Gravity 26, 185005 (2009).

[13] H. Casini, Relative entropy and the Bekenstein bound, Classical Quantum Gravity 25, 205021 (2008).

[14] J. Cardy and E. Tonni, Entanglement hamiltonians in twodimensional conformal field theory, J. Stat. Mech. (2016) 123103.

[15] T. Faulkner, R. G. Leigh, O. Parrikar, and H. Wang, Modular Hamiltonians for deformed half-spaces and the averaged null energy condition, J. High Energy Phys. 09 (2016) 038 .
[16] I. Klich, D. Vaman, and G. Wong, Entanglement Hamiltonians and entropy in $1+1 \mathrm{D}$ chiral fermion systems, Phys. Rev. B 98, 035134 (2018).

[17] R. E. Arias, H. Casini, M. Huerta, and D. Pontello, Entropy and modular Hamiltonian for a free chiral scalar in two intervals, Phys. Rev. D 98, 125008 (2018).

[18] J. J. Bisognano and E. H. Wichmann, On the duality condition for quantum fields, J. Math. Phys. (N.Y.) 17, 303 (1976).

[19] N. Lashkari, Modular Hamiltonian for Excited States in Conformal Field Theory, Phys. Rev. Lett. 117, 041601 (2016).

[20] H. Casini, M. Huerta, and R. C. Myers, Towards a derivation of holographic entanglement entropy, J. High Energy Phys. 05 (2011) 036.

[21] D. D. Blanco, H. Casini, L.-Y. Hung, and R. C. Myers, Relative entropy and holography, J. High Energy Phys. 08 (2013) 060.

[22] T. Faulkner, M. Guica, T. Hartman, R. C. Myers, and M. Van Raamsdonk, Gravitation from entanglement in holographic CFTs, J. High Energy Phys. 03 (2014) 051.

[23] D. L. Jafferis and S. J. Suh, The gravity duals of modular Hamiltonians, J. High Energy Phys. 09 (2016) 068.

[24] A. Belin, N. Iqbal, and S. F. Lokhande, Bulk entanglement entropy in perturbative excited states, SciPost Phys. 5, 024 (2018).

[25] Y. Chen, X. Dong, A. Lewkowycz, and X.-L. Qi, Modular flow as a disentangler, J. High Energy Phys. 12 (2018) 083.

[26] A. Kitaev and J. Preskill, Topological Entanglement Entropy, Phys. Rev. Lett. 96, 110404 (2006). 